ORIGINAL ARTICLE

\title{
Time of physical exercise practice after injury in cervical spinal cord-injured men is related to the increase in insulin sensitivity
}

\author{
JC Koury ${ }^{1}$, MCF Passos ${ }^{2}$, FA Figueiredo ${ }^{1}$, A Chain $^{1}$ and JG Franco ${ }^{3}$
}

Objectives: Physical exercise has an important role in reducing body fat, risk of chronic disease and systemic inflammation. The aim of this study was to determine serum leptin and insulin concentrations and their relationship to the time of physical exercise after injury in men with cervical spinal cord injury (c-SCI).

Methods: $\quad$-SCl subjects with lesion level in C5-C7 $(n=25)$ were divided into two groups: physically active (PA, $n=13$; those who practiced physical exercise for at least 3 months, three times per week or more, for a total minimum of 150 min of physical activity per week) and non-physically active (N-PA, $n=9)$. Body composition was assessed by dual energy X-ray absorptiometry. Blood samples were obtained $12 \mathrm{~h}$ after an overnight fast to measure insulin and leptin in serum, and glucose and C-reactive protein (CRP) in plasma, by validated methods.

Results: Comparing the PA and N-PA group, the first presented lower: total body mass ( $-13 \%)$, body mass index ( $-16 \%)$, fat mass (kg $-39 \%, F M \%-30 \%)$, CRP ( $-23 \%)$, serum insulin ( $-61 \%)$, homeostasis model assessment (HOMA, $-35 \%)$ and serum leptin $(-62 \% ; P<0.05)$. Both serum insulin $(r=-0.561 ; P<0.05)$ and HOMA $(r=-0.591 ; P<005)$ were inversely proportional to the time of practice of physical activity after injury.

Conclusion: Our results suggest that exercise was able to reduce fat mass and increase insulin sensitivity, decreasing plasma levels of risk factors in $\mathrm{c}-\mathrm{SCl}$ subjects.

Spinal Cord (2013) 51, 116-119; doi:10.1038/sc.2012.85; published online 10 July 2012

Keywords: leptin; insulin; physical activity; cervical injury

\section{INTRODUCTION}

Spinal cord-injured (SCI) subjects with reduced activity of the sympathetic nervous system are reported to have an increased risk of cardiovascular morbidity and mortality, variations in leptin and insulin, and modifications in body composition. ${ }^{1}$

The factors such as muscle dysfunction and abdominal obesity may overlap the sympathetic nervous influence. SCI individuals have a reduced concentration of catecholamines and T3, which inhibit lipolysis, favoring the abdominal obesity. ${ }^{2}$ The accumulation of abdominal fat leads to increased serum concentrations of leptin, $\mathrm{C}$-reactive protein (CRP) and insulin resistance.,

Physical exercise has been suggested as an excellent mechanism for the mobilization of adipose tissue and reduced risk of chronic diseases, including people with SCI, possibly due to reduced secretion of leptin and CRP. ${ }^{3,5,6}$ In athletes without lesions, the relationship between serum leptin and body fat is contradictory. Some studies reported that the variation in leptin concentration during exercise was related to variation in fat mass. ${ }^{5,7}$ However, more recent studies revealed that, regardless of body fat mass change, serum leptin levels are different in athletes ${ }^{8,9}$ and in mice. ${ }^{10}$
Previous investigations have shown the beneficial effects of physical training on insulin sensitivity in both healthy and glucose-intolerant subjects. ${ }^{11}$ Hjeltnes et al. ${ }^{12}$ assessed the effect of 8 weeks of electrically stimulated leg cycling in chronic tetraplegics and reported an increase in whole-body insulin sensitivity and an increase in protein expression of key genes involved in glucose metabolism. In agreement with these findings, other studies in chronic SCI individuals demonstrated similar beneficial results of electrically induced cycle training on insulin sensitivity and glucose tolerance. $^{13}$

Based on these reports, we hypothesized that physical exercise may contribute to improvement of hormonal changes in SCI subjects. The main purpose of the present study is to determine the concentration of leptin, insulin, glucose and CRP in subjects with cervical injury. In addition, the association between the time of practice of physical exercise since the injury and glucose homeostasis was obtained. To our knowledge, the relationship between body composition, serum leptin and insulin concentrations and time of practice of physical exercise after injury in cervical SCI (c-SCI) men has not been studied.

${ }^{1}$ Department of Basic and Experimental Nutrition, Institute of Nutrition, State University of Rio de Janeiro, Rio de Janeiro, Brazil; 2 Department of Applied Nutrition, Institute of Nutrition, State University of Rio de Janeiro, Rio de Janeiro, Brazil and ${ }^{3}$ Department of Physiological Science, Biology Institute, State University of Rio de Janeiro, Rio de Janeiro, Brazil

Correspondence: Professor JC Koury, Departamento de Nutrição Básica e Experimental, Instituto de Nutrição, Universidade do Estado do Rio de Janeiro, Rua São Francisco Xavier 524, bloco D - sala 12006, Rio de Janeiro, RJ 20550-900, Brazil.

E-mail: jckoury@gmail.com

Received 3 April 2012; revised 18 June 2012; accepted 19 June 2012; published online 10 July 2012 


\section{MATERIALS AND METHODS}

\section{Subjects}

Twenty-two SCI men ( $33.7 \pm 9.9$ years) participated in this study. Information on lesion characteristics was obtained from medical record. They were quadriplegic and only four subjects presented complete lesion (Table 1). The average duration of injury was $11.6 \pm 8.7$ years. Subjects were matched by sex, age, height, lesion level and duration of injury. According to self-reported information, the most frequent cause of traumatic injury in subjects studied was diving in shallow water (44\%), followed by traffic accidents (40\%) and acts of violence $(12 \%)$. Before the study was conducted, we fully explained its purpose, and obtained written informed consent from each participant. The study protocol was approved by the Research Ethics Committee at the Rio de Janeiro State University (protocol COEP 052/2009).

Data on physical activity was obtained through a structured questionnaire, in which volunteers described the type of physical activity, time spent in total daily physical activity $(\mathrm{min})$ and time of physical activity practice after injury (months). The participants were divided into physically active (PA, $n=13$ ) and non-physically active (N-PA, $n=9)$ groups. Subjects were considered as physically active when practicing regular adapted physical exercise according to the following criteria: total of at least $150 \mathrm{~min}$ per week practiced at least 3 days per week for at least 3 consecutive months. ${ }^{14}$

\section{Anthropometric measurements and body composition}

The height was measured with a stadiometer to the nearest $0.5 \mathrm{~cm}$. From the weight and height values, the body mass index was calculated for each individual using the formula (TBM/ $\left./ \mathrm{HT}^{2}\right)$. Total and regional body mass were measured by dual energy X-ray absorptiometry (DXA; Lunar iDXA with software enCore 2008 version 12.20, GE Healthcare, Madison, WI, USA).

\section{Sample collection}

The subjects were instructed not to eat for $12 \mathrm{~h}$ before sample collection. Blood samples $(10 \mathrm{ml})$ were obtained the morning after ( 0800 hours) by venous puncture into tubes containing heparin as an anticoagulant (30 U per tube) or not. These tubes were centrifuged at $1800 \mathrm{~g}$ for $10 \mathrm{~min}$ for separation of plasma

Table 1 Individual cervical spinal cord injury characteristics

\begin{tabular}{|c|c|c|c|}
\hline Participant & Lesion level & Type lesion & Frankel grade \\
\hline \multicolumn{4}{|c|}{ Physically active } \\
\hline 1 & 7 & Incomplete & C \\
\hline 2 & 6 & Incomplete & C \\
\hline 4 & 5 & Incomplete & $E$ \\
\hline 5 & 5 & Complete & $A$ \\
\hline 6 & 5 & Incomplete & C \\
\hline 7 & 5 & Incomplete & C \\
\hline 13 & 7 & Incomplete & D \\
\hline 18 & 6 & Incomplete & C \\
\hline 20 & 6 & Incomplete & C \\
\hline 21 & 7 & Incomplete & D \\
\hline 23 & 5 & Incomplete & C \\
\hline 24 & 6 & Incomplete & C \\
\hline 25 & 5 & Complete & $A$ \\
\hline \multicolumn{4}{|c|}{ Non-physically active } \\
\hline 3 & 5 & Incomplete & C \\
\hline 9 & 6 & Incomplete & $\mathrm{C}$ \\
\hline 10 & 7 & Incomplete & $\mathrm{D}$ \\
\hline 11 & 5 & Incomplete & $\mathrm{C}$ \\
\hline 12 & 5 & Complete & $A$ \\
\hline 14 & 5 & Incomplete & $\mathrm{C}$ \\
\hline 15 & 5 & Incomplete & C \\
\hline 16 & 5 & Complete & $A$ \\
\hline 17 & 5 & Incomplete & B \\
\hline
\end{tabular}

and at 3000 r.p.m. for $15 \mathrm{~min}$ for obtaining serum, respectively. Blood samples were stored at $-20^{\circ} \mathrm{C}$ until analysis.

\section{Laboratory assays}

Serum leptin was measured through a commercial kit specific for humans (LINCO Research, St Charles, MO, USA; kit reference value from fasting lean body mass index $18-25 \mathrm{~kg} \mathrm{~m}^{-2}$ for men, $\left.3.8 \pm 1.8 \mu \mathrm{gl}^{-1}\right)$. CRP ultra-sensitive (hs-CRP) levels were determined in plasma by immunoturbidimetry (Commercial kit BioClin, Quibasa LTDA (São Paulo, SP, Brazil); the cutoff point to recognize the absence of inflammation was $<0.5 \mathrm{mg} \mathrm{dl}^{-1}$ ) and plasma glucose by enzymatic method (Gold Analyzes, Rio de Janeiro, Brazil; kit reference value was $\left.80-110 \mathrm{mg} \mathrm{dl}^{-1}\right)$. The homeostasis model assessment for the insulin resistance (HOMA2-IR) index was calculated using the HOMA calculator. ${ }^{15}$

\section{Statistics}

Data were presented as mean \pm s.d. Comparison between the groups was performed using the Student's $t$-test. The associations for each group between variables were tested by Pearson correlation matrix. $P$ values $<0.05$ were considered significant. The software used was SPSS 17.0 (SPSS Inc., Chicago, IL, USA) for windows.

We certify that all applicable institutional and governmental regulations concerning the ethical use of human volunteers were followed during the course of this research.

\section{RESULTS}

Table 2 shows the general characteristics of the physically active and non-physically active c-SCI men studied. Age and duration of injury did not differ between the groups. Physical activities practiced by individuals were: wheelchair rugby, touch chair ('wheeling'), swimming and weightlifting.

The PA group individuals had lower total body mass $(P<0.05)$, fat mass (in $\mathrm{kg}, P \leqslant 0.001$ and in percentage, $P \leqslant 0.001$ ) and body mass index $(P<0.01)$ compared with the N-PA group (Table 3$)$.

Table 4 depicts biochemical parameters of the groups. The PA group presents significantly lower serum leptin $(P<0.001)$, serum insulin $(P<0.01)$ and HOMA $(P<0.05)$ compared with the N-PA

Table 2 General characteristics of the physically active and nonphysically active cervical spine cord-injured men studied

\begin{tabular}{lccc}
\hline & $\begin{array}{c}\text { Physically } \\
\text { active }\end{array}$ & $\begin{array}{c}\text { Non-physically } \\
\text { active }\end{array}$ & $\mathrm{P}$ \\
& $\mathrm{n}=13$ & $\mathrm{n}=9$ & \\
\hline Age (years) & $29.6 \pm 8.5$ & $35.2 \pm 11.1$ & 0.191 \\
Duration of injury (years) & $8.7 \pm 7.0$ & $14.2 \pm 10.3$ & 0.162 \\
Time of physical activity practice since the & $30.8 \pm 8.9$ & - & - \\
injury (months) & & - & - \\
Physical activity practice (hours per week) & $12.5 \pm 6.7$ & - &
\end{tabular}

Table 3 Anthropometric measurements and body composition of the spinal cord injury men physically active and non-physically active

\begin{tabular}{lccr}
\hline & $\begin{array}{c}\text { Physically active } \\
\text { Non-physically active }\end{array}$ & $\mathrm{P}$ \\
& $\mathrm{n}=13$ & $\mathrm{n}=9$ & \\
\hline Total body mass (kg) & $64.2 \pm 9.9$ & $73.3 \pm 4.9$ & 0.020 \\
Height (m) & $1.7 \pm 0.1$ & $1.7 \pm 0.1$ & 0.613 \\
Body mass index (kg m $\left.{ }^{-2}\right)$ & $21.2 \pm 2.6$ & $25.1 \pm 3.2$ & 0.004 \\
Fat-free mass (kg) & $45.6 \pm 6.7$ & $44.3 \pm 5.0$ & 0.620 \\
Fat mass (kg) & $16.1 \pm 4.9$ & $26.4 \pm 4.3$ & $\leqslant 0.001$ \\
(\%) & $25.8 \pm 5.7$ & $37.2 \pm 5.1$ & $\leqslant 0.001$
\end{tabular}


Table 4 Hs-CRP, glucose homeostasis and leptin levels of the spinal cord injury men physically active and non-physically active

\begin{tabular}{lccc}
\hline & $\begin{array}{c}\text { Physically active } \\
\mathrm{n}=13\end{array}$ & $\begin{array}{c}\text { Non-physically active } \\
\mathrm{n}=9\end{array}$ & $\mathrm{P}$ \\
& & & \\
\hline Hs-CRP $\left(\mathrm{mg} \mathrm{dl}^{-1}\right)$ & $0.3 \pm 0.6$ & $1.3 \pm 2.7$ & 0.030 \\
Glucose $\left(\mathrm{mg} \mathrm{dl}^{-1}\right)$ & $84.3 \pm 6.2$ & $89 \pm 8.9$ & 0.230 \\
Insulin $\left(\mu \mathrm{ml}^{-1}\right)$ & $8.3 \pm 4.4$ & $13.4 \pm 5.5$ & 0.002 \\
HOMA-IR & $1.1 \pm 0.7$ & $1.7 \pm 0.7$ & 0.030 \\
Leptin $\left(\mathrm{ng} \mathrm{ml}^{-1}\right)$ & $3.5 \pm 1.7$ & $9.2 \pm 2.6$ & 0.001 \\
\hline
\end{tabular}

group. To evaluate the presence of inflammation, the concentrations of hs-CRP were determined, being it higher in the non-active group $(P<0.03)$.

The serum insulin and HOMA were inversely proportional to the time of physical activity practice after injury $(r=-0.56 ; P=0.046$ Figure 1a; and $r=-0.59, P=0.033$ Figure $1 \mathrm{~b}$, respectively).

\section{DISCUSSION}

In the present study, practice of exercise resulted in a significant decrease of total body mass, body mass index and fat mass. The fat mass, both in $\mathrm{kg}$ and percentage, was lower in the group of PA compared with the group of N-PA. The results are related to physical activity, which, admittedly, mobilizes body fat for energy and maintenance of effort including trunk fat, even in this group, which has total immobility of the trunk. ${ }^{16}$ Considering that we did not control the physical activity, we supposed that the difference in body composition in the active group indicates that $150 \mathrm{~min}$ of exercise, practiced at least three times a week for at least 3 months, was sufficient to promote positive changes in body composition in subjects with c-SCI by mechanisms not yet clearly identified.

Our findings show that physically active c-SCI men had lower serum leptin concentration compared with the non-active c-SCI men. Studies about the relationship between leptin and physical activity are contradictory. Pasman et al. ${ }^{7}$ reported that resistance exercise reduces serum leptin, independent of changes in plasma insulin and the percentage of fat mass. Pérusse et al. ${ }^{17}$ suggested that serum concentrations of leptin in humans are not affected due to the acute and/or chronic exercise and are independent of body composition. However, recent studies did not find correlation between serum leptin and fat mass in athletes of different modalities. ${ }^{8-10}$ These authors suggest that the variation in energy intake is responsible for the lack of relationship between fat mass and the secretion of leptin in athletes. Data from these last studies partly corroborate our data from the PA group, as the individuals have SCI.

c-SCI subjects present higher values in serum leptin concentration when compared with the control groups without injury, due to decentralization of the sympathetic nervous system after injury, with increased adipose tissue. ${ }^{18,19}$ The interruption blocks not only the inhibitory effect of sympathetic nervous system on the expression and secretion of leptin but also the stimulatory effect on energy expenditure, and it may lead to the development of hyperleptinemia, decreased metabolic rate at rest and increased adiposity. ${ }^{11,12}$ Probably, the SCI individuals also exhibit leptin resistance due to hyperleptinemia. In the case of the injured who do exercise, leptin may be lower because physical exercise improves leptin resistance via activation of the leptin receptor in hypothalamic nuclei, directly influencing the responsiveness of central nervous system circuits involved in homeostasis energy. ${ }^{10}$ Importantly, despite low levels of leptin in the PA group, these are not correlated with the time of
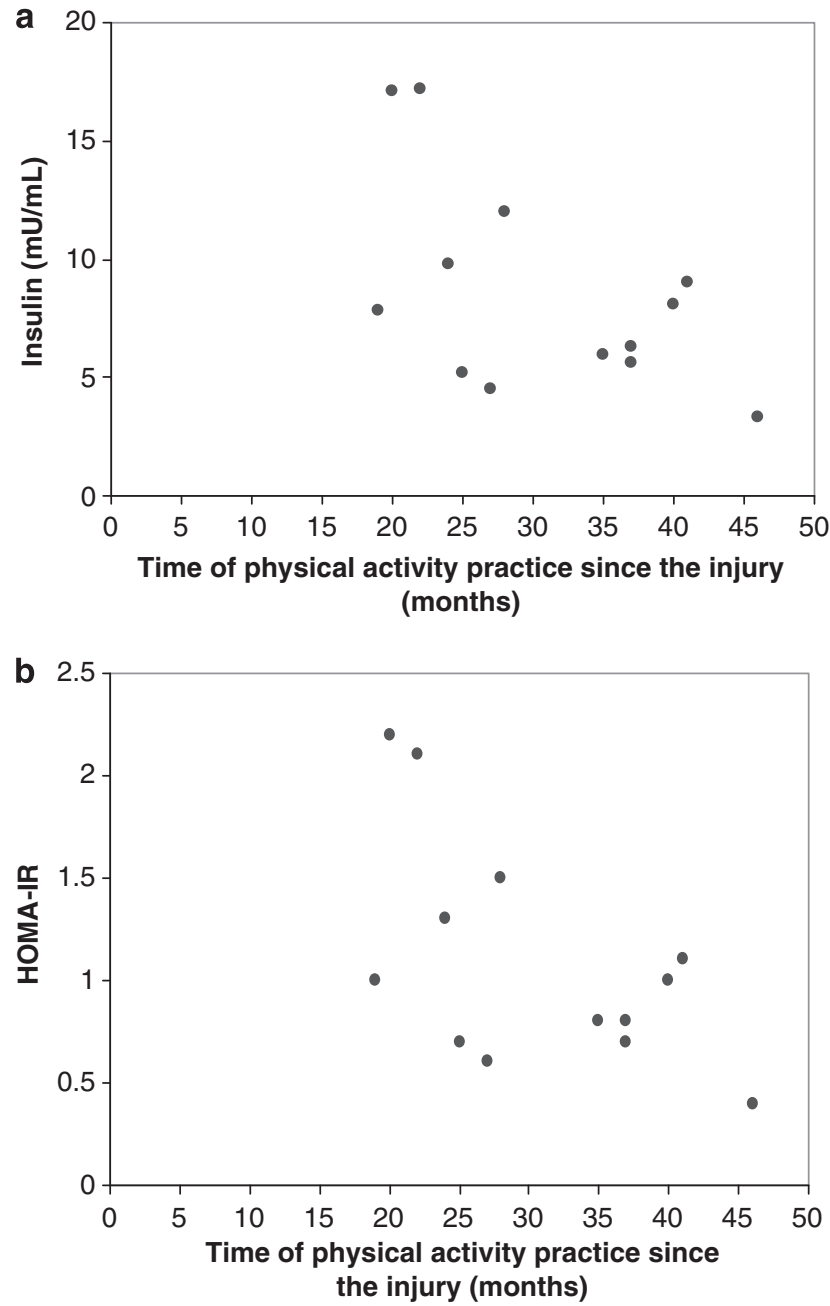

Figure 1 Associations between serum insulin concentration (a; $r=-0.56$ $P=0.046)$ and HOMA ( $b ; r=-0.59, P=0.033)$ with the time of physical activity practice since the injury $(n=13)$.

practice of physical exercise since the injury, differently from the result found with insulin, which will be described below.

The secretion of CRP is directly related to fat mass, and this is an acute-phase protein synthesized by the liver in response to active inflammation. ${ }^{4,6,20}$ Its regulation is related to IL-6, which is either released by adipose tissue or by macrophages. ${ }^{4}$ The ultra-sensitive method was used for the analysis of serum CRP, due to greater accuracy for evaluating the risk of cardiovascular disease. Although both the groups have shown elevated concentrations of CRP, physically active individuals had lower concentrations, suggesting that regular physical exercise can have an important role in preventing cardiovascular disease.

c-SCI individuals are characterized by alterations in glucose intolerance and a decreased whole-body insulin sensitivity. ${ }^{11,21}$ In contrast, in the present study the PA group had lower levels of serum insulin and HOMA compared with the N-PA group. Besides, these parameters were negatively associated with the time of physical activity practice since the injury. Our findings are in agreement with previous studies in SCI individuals, in which an increase in whole-body insulin sensitivity assessed through electrical stimulusassisted cycling training has been reported. ${ }^{12,13}$ The beneficial effects of physical training on insulin sensitivity, in both healthy 
and glucose-intolerant subjects, are well known. ${ }^{2}$ Hence, it seems that physically active SCI individuals have increased insulin sensitivity probably due the physical activity practice.

The mechanism for improved insulin sensitivity in physically active SCI individuals was not established in this study. However, improvements are most likely attributed to changes in muscle characteristics and in the increased capillary density and GLUT- 4 contents. ${ }^{22,23}$ Muscular changes after paralysis, as reduction in the proportion of type 1 and type 2 a fibers, could be one cause of insulin resistance in this population. ${ }^{13}$ Therefore, it is suggested that physical activity reverses these changes in muscle characteristics and GLUT 4 content. Studies showed that electrical stimulus-assisted cycling (8 weeks training/3 or 7 days per week) was associated with increased type 1 and type 2a fibers and increased GLUT-4 and GLUT-1 contents in people with SCI. ${ }^{12,13,22,23}$ No difference between fat-free mass was found in the present study. However, the lower fat mass in PA suggests that physical activity may have improved insulin sensitivity in SCI individuals.

There are two limitations in this study. First, the sample size was relatively small, mainly for non-physically active c-SCI subject. Additionally, we did not monitor physical activity that was reported by the subjects. However, our groups were paired according to the level of lesion (C5-C7), sex, age and the time of lesion duration, all of which being able to affect the results. Despite the evident limitations of the present study, it is of clinical relevance to demonstrate the importance of physical activity to reduce body fat and increase insulin sensitivity, improving plasma levels of risk factors.

In conclusion, the present results indicate that improvements in biochemical and hormonal profile were more pronounced in active c-SCI men. Our results suggest that in physically active SCI men, the exercise was able to reduce body fat with consequent decrease of hsCRP levels and increase insulin sensitivity, performing an important role in preventing cardiovascular disease.

\section{DATA ARCHIVING}

There were no data to deposit.

\section{CONFLICT OF INTEREST}

The authors declare no conflict of interest.

\section{ACKNOWLEDGEMENTS}

We acknowledge the participation of the men who kindly agreed to volunteer for this study. We thank Giselle Louise Cerqueira D'Oliveira and Juliana Esteves Borsatto. This study is part of the program 'Support to construction of the citizenship of people with disabilities,' and is part of the project 'Nutrition and exercise as actions to improve the quality of life, fitness and health of individuals with tetraplegia,' funded by FAPERJ (Foundation for Research Support of the State of Rio de Janeiro), relating to Notice 12/2008 FAPERJProcess and 26/111.788/2008.
1 Maruyama Y, Mizuguchi M, Yaginuma T, Kusaka M, Yoshida H, Yokoyama K et al. Serum leptin, abdominal obesity and the metabolic syndrome in individuals with chronic spinal cord injury. Spinal cord 2008; 46: 494-499.

2 Duckworth WC, Solomon SS, Jallepalli P, Heckemeyer C, Finnern J, Powers A. Glucose intolerance due to insulin resistance in patients with spinal cord injuries. Diabetes 1980; 29: 906-910.

3 De Groot P, Hjeltnes N, Heijboer AC, Stal W, Birkeland KI. Effects of training intensity on physical capacity, lipid profile and insulin sensitivity in early rehabilitation of spinal cord injured individuals. Spinal Cord 2003; 41: 673-679.

4 Beavers KM, Brinkley TE, Nicklas BJ. Effect of exercise training on chronic inflammation. Clin Chem Acta 2010; 411: 785-793.

5 Kraemer RR, Chu H, Castracane D. Leptin and exercise. Exp Biol Med 2002; 9: 701-708.

6 Tsao TH, Hsu CH, Yang CB, Liou TL. The effect of exercise intensity on serum leptin and C-reactive protein levels. J Exer Sci Fit 2009; 7: 98-103.

7 Pasman WJ, Westerterp-Plantenga MS, Saris WH. The effect of exercise training on leptin in obese males. Am J Physiol End Metabol 1998; 274: 280-286.

8 Koury JC, Oliveira KJF, Casimiro-Lopes G, Oliveira AVJ, Portella ES, Moura EG et al. Plasma zinc, cooper, leptin and body composition are associated in elite female judo athletes. Biol Trace Elem Res 2007; 115: 23-30.

9 Esteghamati A, Khalilzadeh O, Rashidi A, Kamgar M, Meysamie A, Abbasi M. Physical activity is correlated with serum leptin independent of obesity: results of the national surveillance of risk factors of noncommunicable diseases in Iran (SuRFNCD-2007). Metabolism 2010; 59: 1730-1735.

10 Krawczewski Carhuanta KA, Demuro G, Tschöp MH, Pfluger PT, Benoit SC, Obici S. Voluntary exercise improves high-fat diet-induced leptin resistance independent of adiposity. Endocrinology 2011; 152: 2655-2664.

11 Dela F, Mikines KJ, Von Linstow M, Galbo H. Effect of training on insulin-mediated glucose uptake in human muscle. Am J Physiol 1992; 263(Part 1): E1134-E1143.

12 Hjeltnes N, Galuska D, Björnholm M, Aksnes AK, Lannem A, Zierath JR et al. Exerciseinduced overexpression of key regulatory proteins involved in glucose uptake and metabolism in tetraplegic persons: molecular mechanism for improved glucose homeostasis. FASEB J 1998; 12: 1701-1712.

13 Mohr T, Dela F, Handberg A, Biering-Sørensen F, Galbo H, Kjaer M. Insulin action and long term-electrically induced training in individuals with spinal cord injuries. Med Sci Spo Exer 2001; 33: 1247-1252.

14 ACSM (American College of Sports Medicine). AHA SUPPORT FEDERAL PHYSICAL ACTIVITY GUIDELINES. U.S. recommendations reflect previously. Available at www.acsm.org/physicalactivity. Accessed 20 March 2009.

15 HOMA Calculator. The Oxford Center for Diabetes. Endocrinology \& Metabolism, Diabetes Trial Unit., Available at http://www.dtu.ox.ac.uk. Accessed 20 December 2011.

16 Goris AHC, Westerterp KR. Physical activity, fat intake and body fat. Physiol Behav 2008; 94: 164-168.

17 Pérusse L, Collier G, Gagnon J, Leon AS, Rao DC, Skinner JS et al. Acute and chronic effects of exercise on leptin levels in humans. J Appl Physiol 1997; 83: 5-10.

18 Huang TS, Wang YH, Chen SY. The relation of serum leptin to body mass index and to serum cortisol in men with spinal cord injury. Arch Phys Med Rehabil 2000; 81: 1582-1586.

19 Wang Y-H, Huang T-S, Liang H-W, Su T-C, Chen S-Y, Wang T-D. Fasting serum levels of adiponectin, ghrelin, and leptin in men with spinal cord injury. Arch Phys Med Rehabil 2005; 86: 1964-1968.

20 Frost F, Roach MJ, Kushner I, Schreiber P. Inflammatory C-reactive protein and cytokine levels in asymptomatic people with chronic spinal cord injury. Arch Phys Med Rehabil 2005; 86: 312-317.

21 Hjeltnes N, Aksnes AK, Birkeland KI, Johansen J, Lannem A, Wallberg-Henriksson H. Improved body composition after $8 \mathrm{wk}$ of electrically stimulated leg cycling in tetraplegic patients. Am J Physiol 1997; 273: R1072-R1079.

22 Chilibeck PD, Bell G, Jeon J, Weiss CB, Murdoch G, MacLean I et al. Functional electrical stimulation exercise increases GLUT-1 and GLUT-4 in paralyzed skeletal muscle. Metabolism 1999; 48: 1409-1413.

23 Lillioja S. Skeletal muscle capillary density and fibre type are possible determinants of in vivo insulin resistance in men. J Clin Inv 1987; 80: 415-424. 